\title{
Distance Assisted Information Dissemination with Broadcast Suppression for ICN-based VANET
}

\author{
Yuhong $\mathrm{Li}^{1}$, Xiang $\mathrm{Su}^{2}$, Anders Lindgren ${ }^{3,4}$, Xinyue Shi ${ }^{1}$, Xiang Cai ${ }^{1}$, Jukka Riekki ${ }^{2}$ \\ ${ }^{1}$ State Key Laboratory of Networking and Switching Technology \\ Beijing University of Posts and Telecommunications, China \\ ${ }^{2}$ Centre for Ubiquitous Computing, University of Oulu, Finland \\ ${ }^{3}$ SICS Swedish ICT, Sweden \\ ${ }^{4}$ Luleå University of Technology, Sweden
}

\begin{abstract}
Information-centric networking (ICN) is being applied to the vehicular networks by more and more researchers on account of its lightweight and connectionless networking paradigm and in-network caching characteristics, making it suitable for the dynamic environments of vehicular networks. However, wireless transmission of interest packets to find content in the network may lead to broadcast storms that can affect the performance of information dissemination severely. This paper proposes a distance assisted data dissemination method with broadcast storm suppressing mechanism (DASB) for supporting rapid and efficient information dissemination in ICN-based vehicular ad hoc networks (VANETs). Geo-position data of vehicles are used to accelerate packet forwarding, and vehicular nodes in certain areas are restricted to forward packets in order to suppress the broadcast storm. Simulation results show that the proposed method can greatly reduce the total number of packets transmitted in the network, and the successful information delivery ratio and information delivery time can also be improved.
\end{abstract}

Keywords. Information-centric networking, VANET, Broadcast storm suppression.

\section{$1 \quad$ Introduction}

By connecting vehicles to people, other vehicles, and their surrounding environment, data about road and environmental conditions can be obtained by any vehicle and personal and entertainment offerings can be provided to passengers. To that end, vehicular ad hoc networks (VANETs) have been regarded not only as a key technology for increasing road safety and transport efficiency, and providing infotainment for people in mobile environments, but also as a method for increasing revenue for service providers and car manufacturers. Nevertheless, due to the highly dynamic mobile environment and the resulting wireless transmission conditions, some problems still need to be solved in order to bring VANET into reality. 
Information-centric Networking (ICN) is a novel networking paradigm [1] that does not require global IP address allocation and management, setup of data delivery paths, or session establishment and management. Focus in the network is moved from named end-points to distribution of named data from producers or intermediate caches in the network. Thus, many major requirements of VANETs can be matched by the ICN paradigm. For example, the receiver-driven and name-based data delivery mode reduces the high overhead of mobility management and session maintenance. Asynchronous ICN communication suits the intermittent links of VANETs well. The in-network caching mechanism of ICN provides help to reduce the latency of obtaining data by vehicles, in particular for data of limited geographic interest. Additionally, most applications in VANET are information oriented and in many cases, vehicles and people on board do not need to directly interact with each other, but are satisfied with obtaining the needed information, and do not mind who provides the information and how and from where it comes from. At the same time, the computing and storage resources required by ICN, such as name-based data forwarding and in-network caching can be provided at low cost by vehicles which do not have restrictions on battery size, volume, or weight that many other mobile devices suffer from. Hence, the ICN paradigm is a suitable candidate for realizing VANETs.

However, introducing ICN in VANET also faces challenges. We address a typical VANET using wireless transmission technology such as IEEE $802.11 \mathrm{p}$, where broadcasting data over a network interface is the basic communication mode between two nodes. We assume that nodes make use of the possibility to overhear packets to facilitate efficient multicasting of Interest packets such that they can spread quickly throughout the network. However, in the receiver-driven ICN paradigm, if an information request (e.g., the Interest packet in NDN [2]) is broadcasted, multiple copies of the request will be transmitted through the network, with the risk for routing loops and other redundant transmissions. When in-network caching mechanism is used, multiple copies of the responded data will also be produced and spread over the network. This not only wastes the valuable wireless transmission resources, but also, due to interference, decreases transmission reliability. Moreover, the density of the vehicle nodes and the applications running in the vehicles may change heavily and frequently, leading to unbalanced data traffic. Hence, a broadcast storm may heavily influence the quality of data transmission. In addition, the inherent feature of VANET, that is, short-lived intermittent connectivity, reduces the possibility of data dissemination. As a result, all vehicular nodes having transmission opportunity take part in the data forwarding in most routing mechanisms [3][4]. This makes the problem of broadcast storm even worse.

In this paper, we introduce DASB, a distance assisted data dissemination method with a broadcast storm suppression mechanism, to realize efficient and fast data dissemination in ICN-based VANETs. Our work aims at making the ICN paradigm viable for VANETs by designing an efficient data dissemination mechanism with low packet redundancy. Specially,

- We propose a packet forwarding strategy to reduce the numbers of hops of packet forwarding and at the same time to suppress the forwarding of the nearby nodes, by making use of the geo-position information of the vehicles;

- We introduce a mechanism to suppress the broadcast storm while keeping the 
successful packet delivery ratio by limiting the amount of nodes around the current forwarding node that broadcast the data further;

- We evaluate the proposed mechanisms through implementation and carrying out simulations. Especially, we use real world traffic and a vehicle mobility model to validate the proposed mechanism.

The rest of the paper is organized as follows. In Section II, the distance assisted packet forwarding scheme for ICN-based VANETs is described. In Section III, the method of broadcasting suppression and the corresponding suppression angle are introduced. Following this the implementation and simulation environment are described, and the performance of the proposed method is evaluated and discussed in Section IV. We discuss the related work in Section V. Finally, Section VI concludes the paper.

\section{Distance Assisted Packet Forwarding}

Work in [5] [6] [7] has investigated the advantages of introducing information centric networking into VANETs, and made some improvements to the basic NDN architecture to implement the name-based data dissemination in VANETs. In short, we borrow ideas from these works and keep the following functions: (i) packets are allowed to be broadcast over the same wireless network interface from which the packets were received; (ii) the CS (Content Storage) and PIT (Pending Interest Table) functionality remains as for normal NDN operation; (iii) a timer based mechanism that allows forwarding of packets after waiting a certain time is used in each intermediate node.

A problem with previous solutions is that the mechanisms proposed have the potential to create broadcast storms in the network, leading to high overhead and waster of network resources. In order to suppress such broadcast storms, we reduce the data transmitted in the network, and therefore improve data dissemination efficiency. We introduce the following features in our system: (i) the data structures Forwarded Table (FT) and Waiting Table (WT) in the nodes; (ii) a modified format of Interest and Data packets, carrying geo-position information; (iii) a method for calculating the retransmission deferral timer and packet forwarding method based on internode distance; and (iv) a broadcast storm suppression mechanism. The first three features are described below, and the fourth feature will be explained in Section III.

\subsection{Packet forwarding according to distance}

All data is transmitted through broadcasting it over the wireless network interface. Since many nodes are able to receive the packets by overhearing the wireless channel, this allows Interest and Data packets to be forwarded by intermediate nodes to increase the likelihood of reaching the destination, but this can also lead to loops and repeated forwarding. This may in turn cause heavy packet loss due to serious interference in the physical layer and network layer congestion.

To avoid loops and repeated forwarding of Interest packets, we introduce a Forwarded Table (FT) in each node which records the Nonce, a unique identifier used when creating an Interest, and the forwarding time of the Interest by the current node. The table is subsequently used to ensure that an Interest packet is forwarded at most 
once. A node frees table entries according to a local strategy, for example, after a certain time, when it can be assumed that possible local forwarding of the packet in the neighbourhood has ceased.

To shorten the data response time and to reduce the amount of packets transmitted in the network, we use a greedy geo-position based forwarding strategy. Namely, we allow the vehicular nodes furthest away from the current node to forward the packet first. This is because that node will propagate the packet the furthest physical distance and thus is likely to bring the packet closer to a remote destination that is not in range of the first node. In this way, fewer vehicular nodes are involved in packet forwarding, and fewer hops between the content requestor and responder are needed. This can be expected to decrease the latency of requesting content and also the interference and therefore also improve the content delivery ratio.

In order to be able to estimate the distance between the previous node that broadcasts the packet and the nodes receiving the packets by overhearing the wireless channel, we modify the original Interest and Data packets in NDN to include geo-position information of the previous node, as shown in Fig. 1. Thus, when a node receives, for example, an Interest packet, it can calculate the distance between itself and the previous forwarder, according to its own position information and the position information carried in the packet. In vehicular networks, it is common that all vehicles have a GPS navigator, so including the location of the node in the message is a feasible requirement.

\begin{tabular}{|l|l|l|l|l|}
\hline Name & Selectors & Nonce & Guiders & PreNode_position \\
\hline
\end{tabular}

Fig. 1. Format of Interest packets

In order to enable the furthest node to forward the packet without knowing the distance information of other nodes, we introduce two types of retransmission deferral timers, $T_{i}$ and $T_{d}$, in each vehicle node. When a node receives an Interest packet that is not listed in its FT, it calculates the distance to the previous node that sent the packet. Then it sets the retransmission deferral time for timer $T_{i}$ according to a "waiting time function", where the value of the timer is dependent on the distance, and adds the packet information to its Waiting Table (WT). The longer the distance, the shorter the deferral time. As a result, the timer in the node furthest away will expire first, and the node will forward the Interest first. During the deferral time of $T_{i}$, all the nodes listen for other transmissions of the same Interest packet. Upon overhearing the same Interest transmitted by another node, the nodes will discard the Interest and stop the timer $T_{i}$. The same mechanism is applied when a Data packet is received by a node, using timer $T_{d}$.

\subsection{Waiting time function and waiting table}

Considering the case that multiple vehicle nodes may have the same distance to the previous node, the deferral time $t$ consists of two parts $t=t_{1}+t_{2}$, where $t_{1}$ is the time related to the distance, $t_{2}$ is a random time, and $t_{2}<<t_{1}$ (i.e., $t_{2}$ is far smaller than $t_{1}$ ). Thus, one of the nodes having the longest distance will have the smallest $t$, and will forward the Interest or Data first, by broadcasting it through the wireless network interface. 
The longest distance for a vehicle being able to forward packets is determined by the maximum distance of wireless transmission. In order to determine the deferral time for each node, we assume:

$R$ is the reliable radio transmission distance. For simplicity, here we suppose all the vehicles have the same $R$.

$D_{m}$ is the longest distance between two vehicles to send and receive data, i.e., no data transmission errors are caused by the distance. $V_{m}$ is the maximum velocity of the vehicles and $L_{m}$ is the maximum tolerated latency for one hop. Considering the movement of the vehicles $D_{m}=R-2 V_{m} \times L_{m}$, which is the case that two vehicles travel to the opposite directions at the maximum speed.

The deferral time is a decreasing function of the distance, and depending on the geographical distribution of the vehicular nodes in the network, different functions can be used. In this paper, we assume that vehicles are randomly distributed. We assign a simple linear decreasing function to the deferral time, as shown in Fig. 2.

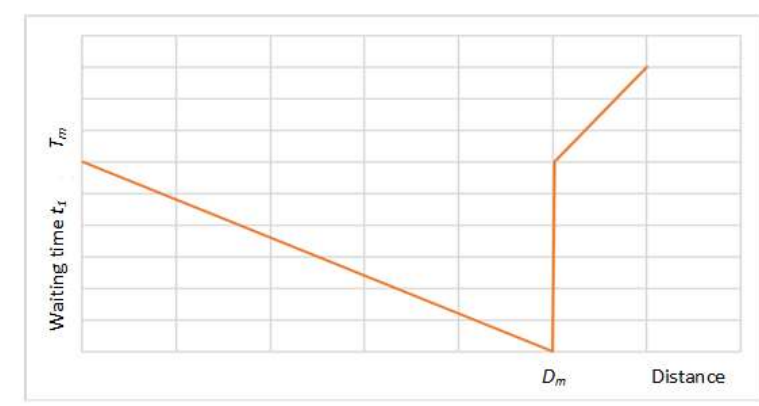

Fig. 2. Deferral time function

The maximum time $T_{m}$ when the distance is 0 may eventually have influence on the data response latency. If it is too big and the network density is lower, vehicular nodes nearby the forwarding node may have relatively long deferral time. Theoretically, $T_{m}$ should be $T_{\text {hop }}<T_{m}<2 T_{\text {hop }}$, where $T_{\text {hop }}$ is the maximum transmission and processing delay of one hop. In practice, the maximum time can be determined by considering the average density of the vehicles in selected scenarios and the accuracy of the clocks. Fig. 8 in Section IV illustrates the relationship between $T_{m}$, the density of the vehicular nodes and the data response latency obtained by our simulations.

It should be noted that the synchronization of clocks among vehicles will not play a role in our method because the deferral time is used and it only requires measuring relative time intervals.

\section{$3 \quad$ Forwarding suppression}

\subsection{Forwarding suppression angle}

As shown in Fig. 3, when an Interest is sent by node A, both node B and node $\mathrm{C}$ will receive the packet. Since the distance from $\mathrm{B}$ to $\mathrm{A}\left(d_{B A}\right)$ is longer than that from $\mathrm{C}$ to $\mathrm{A}$ 
$\left(d_{C A}\right)$, B will forward the packet before C. In other words, C's forwarding is suppressed. However, if node D has the requested content, as shown in Fig. 3, the content can be obtained only when node $\mathrm{C}$ forwards the Interest. Here we assume that $\mathrm{D}$ is the nearest node having the requested information when multiple copies of the requested information exist. To avoid forwarding a packet far away from the content holder, when the position of such a node D is known, we introduce a suppression angle $\alpha_{S A}$, to determine a sector within which packet forwarding is suppressed. In the case presented in Fig. 3, node $\mathrm{C}$ is outside this sector and hence forwards the packet and node $\mathrm{B}$ is the only node inside the sector forwarding the packet.

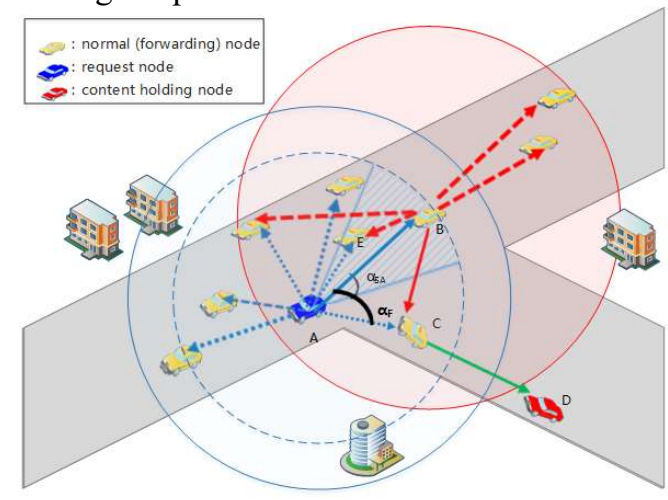

Fig. 3. Forwarding suppression angle

As shown in Fig. 3, after node $\mathrm{C}$ overhears the Interest from $\mathrm{B}$ during the deferral time, the angle $\alpha_{F}$ formed by line $\mathrm{AB}$ and $\mathrm{AC}$ can be calculated, namely:

$\alpha_{F}=\arccos \frac{d_{B A}{ }^{2}+d_{C A}{ }^{2}-d_{C B}{ }^{2}}{2 d_{B A} \cdot d_{C A}}$. Here, $d_{B A}, d_{C A}, d_{C B}$ are the distances between node $\mathrm{B}$ and $\mathrm{A}$, node $\mathrm{C}$ and $\mathrm{A}$, and node $\mathrm{C}$ and $\mathrm{B}$, respectively. These distances can be calculated when node $B$ and $C$ receive the Interest from node $A$ and when $C$ receives the same Interest from $B$ again. In this case, node $C$ will first calculate the angle $\alpha_{F}$ according to the geo-position information carried in both Interest packets. If $\alpha_{F}<\alpha_{S A}$, it means that $\mathrm{C}$ is in a direction similar enough to $\mathrm{B}$, and since $\mathrm{B}$ is expected to be closer to the content holder, $\mathrm{C}$ will discard the Interest received both from B and A, stop the deferral timer, and delete the corresponding entry from its waiting table WT. In This way, redundant Interest packet forwarding will be suppressed, but the forwarding of the Interests will not be restricted only to a certain direction, all directions may receive the Interest. If multiple copies of requested information are cached in the network, the suppression of Interests will greatly reduce the amount of the responded Data packets. Similarly, certain amount of Data packets will also be suppressed.

\subsection{Suppression angle and information delivery ratio}

Since the successful delivery of an Interest packet to a node holding the requested information is related to the suppression angle $\alpha_{S A}$, we calculate the maximum failure probability of forwarding the Interest as a function of $\alpha_{S A}$. 
We start by re-drawing the situation described in Fig. 3 as Fig. 4. To study the situation in which an Interest is not forwarded, we place point $D$ at the intersection of circles $\mathrm{A}$ and $\mathrm{B}$. They all have the same radius $R$, which denotes the radio transmission distance of the vehicular nodes A and B. $d_{A B}$ is the distance between $\mathrm{A}$ and $\mathrm{B}$. As shown in Fig. 4, the following situation will lead to a cessation of packet forwarding: (i) there are no nodes in the grid area; (ii) nodes exist in the stripe area, in other words, there are nodes that can receive the forwarded packets; (iii) all nodes that received the forwarded packets are distributed in the suppressed area. In this case, all the nodes that have the possibility to forward packets further are suppressed.

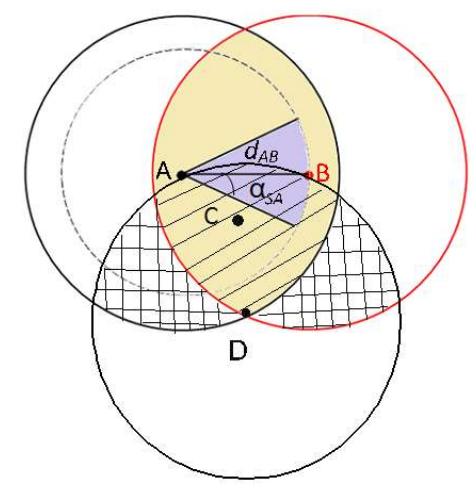

Fig. 4. Suppression angle and probability of packet loss

Suppose the vehicles are randomly distributed in the considered area with an average density of $\rho$. Then the probability of the above condition (ii) is $P(D)=$ $\frac{4 \pi-3 \sqrt{3}-6 \sin ^{-1} \frac{d_{A B}}{2 R}}{5 \pi-3 \sqrt{3}}$. The probability of the above condition (iii) is $P(C)=\frac{6 \alpha_{S A} d_{A B}{ }^{2}}{(4 \pi-3 \sqrt{3}) R^{2}}$. Suppose $m, n$ are the number of nodes in the grid and the stripe area respectively, therefore, $m=\rho \times\left(\frac{\pi}{6} R^{2}+\sin ^{-1} \frac{d_{A B}}{2 R} \cdot R^{2}\right) ; n=\rho \times\left(\frac{2}{3} \pi R^{2}-\frac{\sqrt{3}}{2} R^{2}-\sin ^{-1} \frac{d_{A B}}{2 R} \cdot R^{2}\right)$.

Thus, the probability of failing forwarding is $P=P(D)^{m} \cdot P(C)^{n}$. For example, when $d_{A B}=R, \alpha=\frac{\pi}{3}, \rho=9 / \pi R^{2}, m=3, n=2 ; P=4.7 \%$; and when $d_{A B}=R$, $\alpha=\frac{\pi}{6}, \rho=9 / \pi R^{2}, m=3, n=2 ; P=1.1 \%$. The relationship among suppression angle, the delivery ratio of Interest packets and the density of vehicular nodes is shown in Fig. 9.

\subsection{Packet processing at vehicular nodes}

A node wishing to retrieve data from the network broadcasts an Interest packet expressing its wish through the name of the requested data. The processing at an intermediate node is as follows. As shown in Fig. 5 (a), each node receiving an Interest ("new instance") first checks if the same Interest ("old instance") is already in the Waiting Table (WT) matching the Nonce field of the Interest. If yes, the node next decides if the Interest should be suppressed based on the forwarding angle $\alpha_{F}$ of the new instance and the suppressing angle $\alpha_{S A}$. If $\alpha_{F}>\alpha_{S A}$, which means the node is in a location different 
from the "new" previous node (i.e., Node B in Fig. 3), it will continue running the old deferral timer, until it times out and forwards the Interest (i.e., the Interest it received earlier from node A in Fig. 3). If $\alpha_{F}<\alpha_{S A}$, the node will stop the old timer, delete the corresponding entry in the WT and discard the Interest (e.g. node E in Fig. 3).

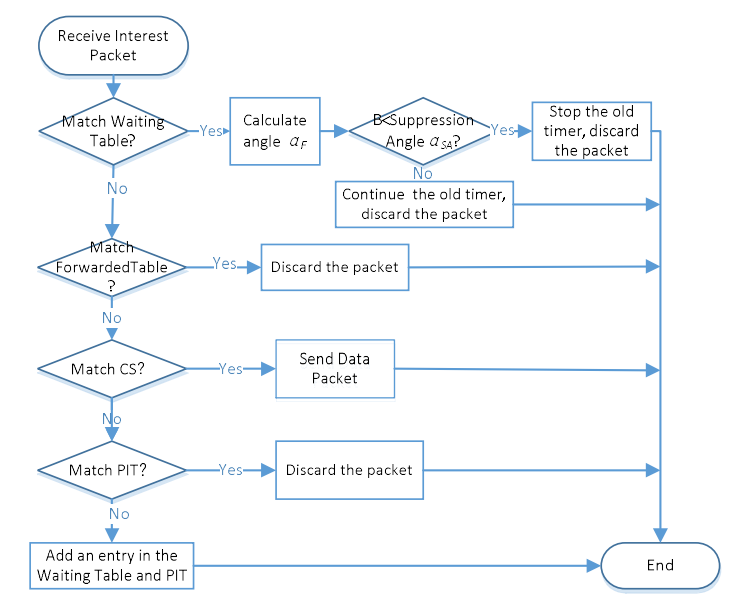

(a) Processing of Interest Packet

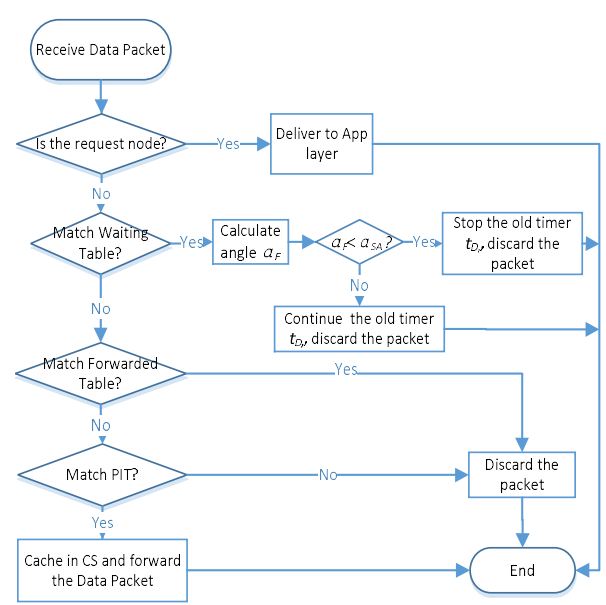

(b) Processing of Data Packet

Fig. 5. Processing of Interest Packet and Data Packet

If no entry is found in the WT, the node will check if the Interest is in the Forwarded Table. If yes, the packet is discarded. Otherwise, the node searches the information name in its CS, similarly to the original NDN mechanism. If a match is found, the node starts the Data response procedure. If not, it will check if there is already an entry exist in the PIT. If a match is found, the node will not forward the Interest, since the same Interest has been forwarded already. Otherwise, the node will add an entry in the WT 
by initiating a new timer $t_{I}$ according to the waiting time function and register it in the PIT. The node will schedule the Interest forwarding when the timer expires.

Similar processing is used when a Data packet is received at a node, except caching is done at the intermediate node, as shown in Fig. 5 (b).

\section{Performance Evaluation}

We implemented the proposed method in ndnSIM [8], which realizes the basic mechanisms of NDN in the network simulator ns-3 [9]. We extended the ndnSIM implementation with support for the functionality described in this paper (Forwarded Table, Waiting Table, waiting time function and the corresponding broadcast suppression method).

To evaluate the performance of the proposed method, we use the tool OpenStreetMap (OSM) [10] and Simulation of Urban Mobility (SUMO) [11] to create different mobility scenarios. OSM provides freely exportable maps of cities. The OSM road information is generated and validated by satellite images and GPS traces, and is commonly regarded as the highest quality road data publicly available today. SUMO is an open-source traffic simulator with continuous space and discrete time. SUMO is capable of importing maps in multiple formats, including OSM. In our simulation, we use OSM to create the real-world map, and use SUMO to generate vehicle nodes and traffic, and mobility model in the real-world road maps.

We evaluate the proposed method using the metrics total number of messages transmitted in the network, data request and response (i.e., round-trip) time and successful information delivery ratio by comparing our approach with basic NDN. Fig. 6 shows the parameters of ndnSIM used in our simulations.

\begin{tabular}{|l|l|}
\hline Parameters & Value \\
\hline WLAN Protocol & IEEE 802.11p \\
\hline Data Link Types & DLT_IEEE802_11_RADIO \\
\hline Wireless transmission model & ConstantSpeedPropagationDelayModel \\
\hline Signal propagation loss model & RangePropagationLossModel \\
\hline Receiving gain & $0 \mathrm{db}$ \\
\hline Remote station manager & ConstantRateWifiManager \\
\hline Data transmission rate & 10 packets/s \\
\hline Time & $50 \mathrm{~s}$ \\
\hline
\end{tabular}

Fig. 6. Parameter set during simulations

Fig. 7 illustrates the map obtained by using OSM and imported in SUMO. This is the map of Solna in Stockholm, Sweden, with an area of about $8.05 \mathrm{~km}^{2}$. We measure the performance of the proposed method when there are 100, 200, 400 and 600 vehicles respectively distributed in the sections of roads randomly. In our tests, $10 \%$ of the nodes send Interests with different names (i.e., asking for different content) every 0.5 seconds. The test time is 10 seconds. Another $10 \%$ of the nodes serve as content providers, holding the requested content and are randomly distributed in the network. In order to analyse the effects of the distance assisted routing and broadcast suppression, we disabled caching in the intermediate nodes. We assume that the nodes are able to know their own 
position and for evaluation of broadcast suppression, we also assume that nodes know the position of the content provider.

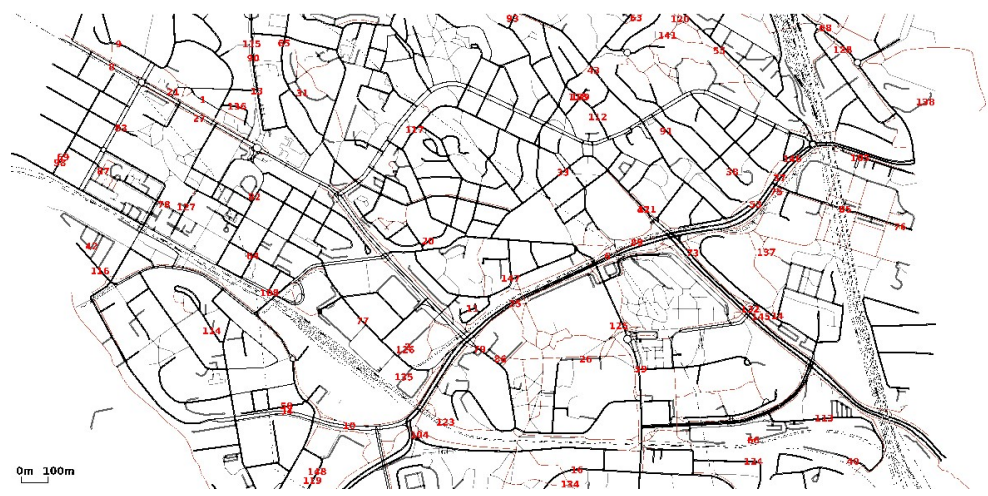

Fig. 7. Map for the simulations

\subsection{Results}

To evaluate the influence of the maximum deferral time $T_{m}$ to the latency of packet forwarding, we measure the average round-trip latency of information requests with different $T_{m}$ values. Here, we measure latency as the elapsed time from sending an Interest packet until receiving a Data packet as response under different vehicular densities in the network. Fig. 8 illustrates the results. Here we can see that with the increase of $T_{m}$, the round-trip latency increases. This is because each forwarding node will wait a relatively longer time on average when $T_{m}$ increases. However, with the increase of the number the nodes in the fixed area, the distance between two nodes becomes shorter, the average deferral time of each forwarding node becomes longer, and therefore the round-trip latency increases. However, the distance-based routing does not increase the number of hops of data forwarding when the number of vehicles increases and thus, the round-trip latency will not increase in general.

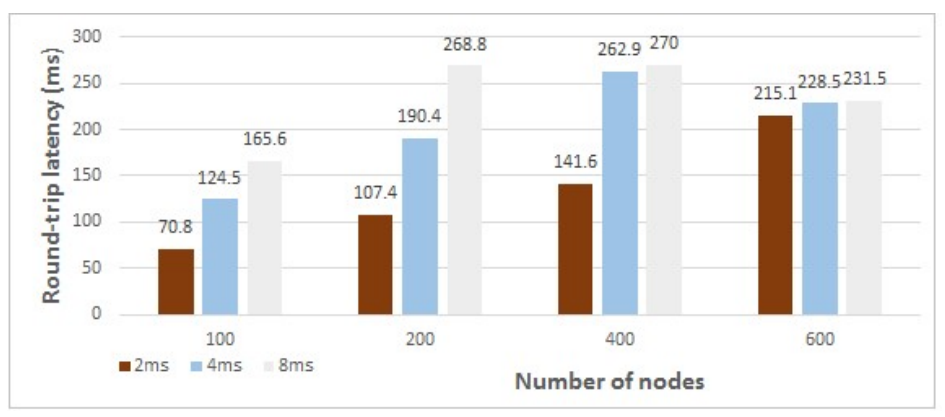

Fig. 8. Relationship among round-trip latency, Tm and node density

Fig. 9 illustrates the relationship between suppression angle, information delivery ratio and density of vehicular nodes. Here the delivery ratio is defined as $D_{r}=N_{\text {sent }} / N_{\text {rec }}$, 
where $N_{\text {sent }}$ is the total number of Interest packets sent by nodes in the network and $N_{\text {rec }}$ is the total number of received Data packets requested by the nodes. We can see that increasing the suppression angle will result in the reduction of the information delivery ratio when the density of the vehicles is fixed. In addition, with the increase of the density of vehicles, the information delivery ratio may decrease further. This is because more vehicles are in the radio transmission area, therefore there will be more nodes to take part in the packet forwarding, and there will be more congestion and more packet loss. However, by using our proposed method, the delivery ratio decreases not as radically as the basic NDN method and it can be seen that it is at higher node densities that the suppression of broadcasts become more important.

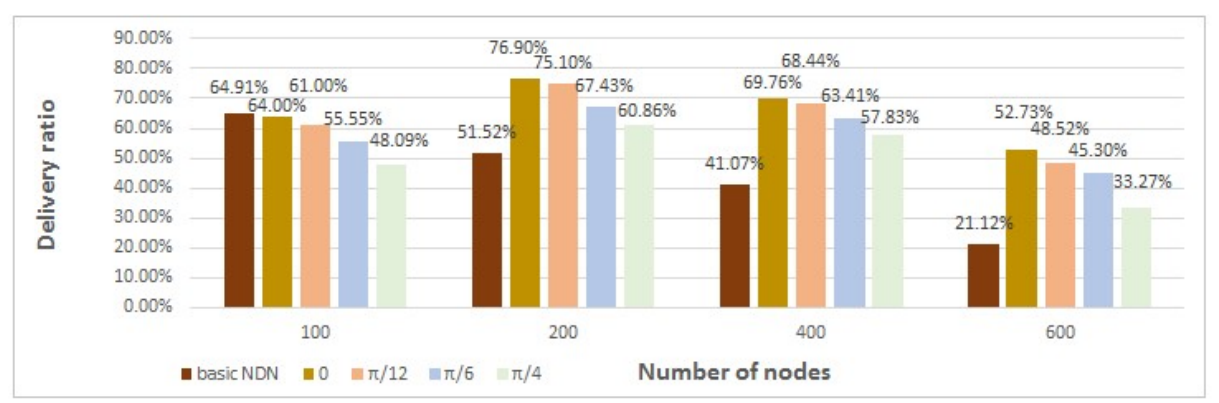

Fig. 9. Relationship among suppression angle, delivery ratio and node density

Fig. 10 illustrates the total number of Interest and Data packets transmitted in the network with different node density (i.e., different number of nodes in the fixed area) compared with the basic NDN mechanism. Note that all the requested information are different, and there is only one copy of each information in the network. In other words, no caches of information are available. We compare also the effects when different suppression angles are used. The total amount of traffic in the network increases with a higher number of nodes since that corresponds to a larger number of content requests in our model, but we can see that the same trend holds true for all node densities. Our broadcast suppression mechanisms decreases the number of packets radically, reducing the traffic volume with $70-97 \%$ depending on node density, when compared with the original NDN method. In addition to the general gain in delivery ratio that we could see in Fig. 9, this also means that the overhead and resource usage for each piece of delivered content is significantly lower, leaving room for other traffic in the network. 


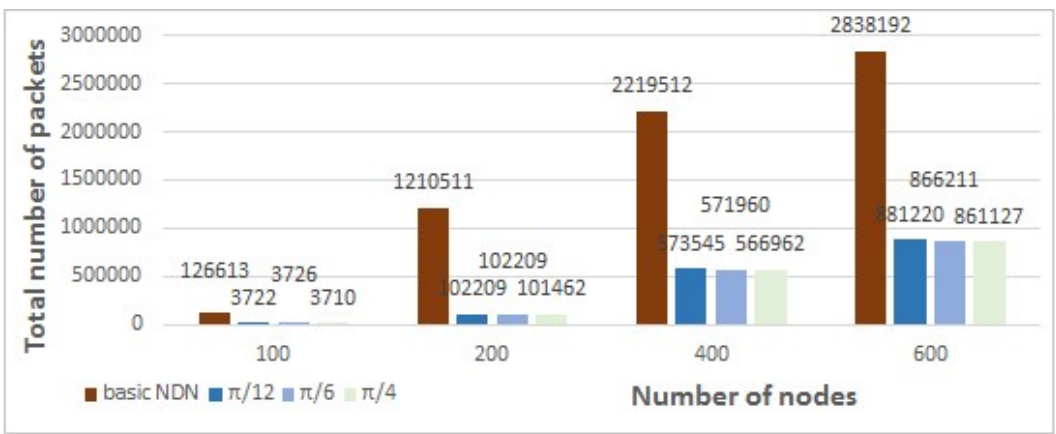

Fig. 10. Number of packets forwarded in the network

Fig. 11 illustrates the average latency for obtaining data compared with the basic NDN mechanism, i.e., the time between an Interest is sent and the first Data packet is received. Here we can see that in general our proposed method performs comparable to basic NDN at very low or high node densities and has over 30\% shorter latency for obtaining information than the basic NDN method in medium-density scenarios.

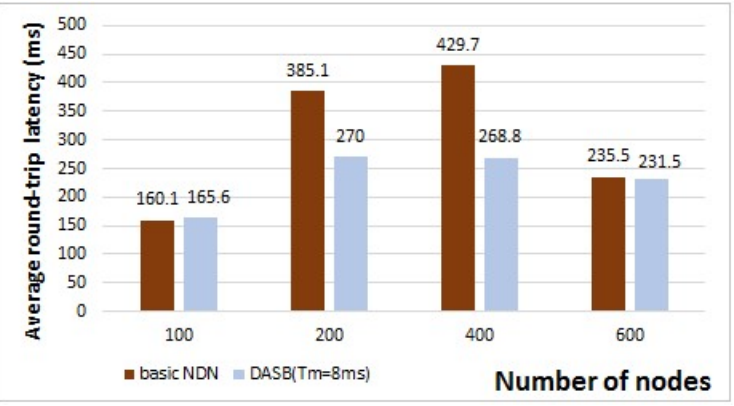

Fig. 11. Average latency of information request and response

\section{$5 \quad$ Related Work}

VANET and MANET (Mobile ad hoc networks) share the same principle not relying on fixed infrastructure for communication. They have many similarities, such as self-organization, low bandwidth and short range of radio transmission. Hence, most ad hoc routing protocols are still applicable for VANET, such as AODV (Ad-hoc Ondemand Distance Vector) [12] and DSR (Dynamic Source Routing) [13] etc. However, VANET differs from MANET in its highly dynamic topology. A number of studies [14] [15] [16] have been have shown that most ad hoc routing protocols (e.g., AODV and DSR) suffer from highly dynamic nature of node mobility. Therefore, some routing protocols have been presented for VANET, including position-based routing [15] [17], broadcast routing [18] and cluster-based routing [17] [19].

Among them, GPSR (Greedy Perimeter Stateless Routing) [20] is one of the most known position-based protocols. It combined the greedy routing with face routing by 
using face routing to get out of the local minimum where greedy fails. It works best in a free open space scenario with evenly distributed nodes. In this paper, we use geographical location information and greedy algorithm in the context of ICN, since vehicular nodes' movement is normally constrained along roads and streets. Geographical information can be easily obtained from street maps and modern navigation systems. In addition, by using the greedy methods, packets suffers less latency in general.

Broadcast is a frequently used routing method in VANETs. Broadcast is also used in unicast routing protocols during the routing discovery phase to find an efficient route to the destination. The simplest way to implement a broadcast service is flooding, where each node broadcasts message to all its neighbours except the one from which it got this message from. Through flooding the message will eventually reach all nodes in the network. Flooding performs relatively well when there are a certain small number of nodes in the network and is easy to be implemented. However, when the number of nodes in the network increases, the bandwidth needed for one message transmission may increase exponentially. In the wireless environment, this may also cause heavy contentions and collision. Thus, the performance of transmission will decrease heavily. Flooding may has also a significant overhead. In this paper, the flooding mechanism is used to deliver the messages in the context of ICN, and we concentrate on reducing the number of broadcasted messages in order to reduce the overhead and increase the bandwidth usage.

Several researchers have investigated the possibilities of using the ICN paradigm in VANETs. For example, the ICN Research Group of the Internet Research Task Force (IRTF) has identified vehicular networks as a key scenario in which ICNs are likely to play an important role and outlines some challenges and existing work in the area in [21]. In [5] and [6], authors report studies on the advantages of using Named Data Networking (NDN) [22] in vehicular networks. Here, the focus has been on introducing basic NDN mechanisms in VANETs, such as where and how to implement NDN concepts like the PIT (Pending Interest Table), and CS (Content Store) and how to combine it with flooding mechanisms to distribute data. Work reported in [7] takes this one step further. A counter-based broadcast scheme and two transmission defer timers are introduced to counteract the broadcast storm phenomena, by reducing packet collision events on the wireless medium. However, no advanced mechanism is presented to suppress the broadcast storm. [23] and [24] present methods for Interest forwarding in NDN. We borrow from V-NDN [23] the idea of using a simple greedy forwarding method to spread Interest. However, instead of spreading Interests to all directions, we restrict the spreading of Interest in certain area to suppress the broadcasting storm. Navigo in [24] coupled names with the locations where the data reside: Interests are either forwarded according to the FIB with a probability $\mathrm{p}$ or simply flooded with a probability of 1-p, in order to avoid the packets focusing on a single destination area for a long time. However, the purpose of the mechanism is to balance the traffic among different sources, not suppress broadcast storm. In addition, both [23] and [24] discuss only forwarding Interests, forwarding the responses carrying the requested data is not discussed. The mechanism proposed in this paper applies to forwarding of both Interest and Data packets. 


\section{Conclusions and Future Work}

An information dissemination method for ICN-based VANETs has been proposed in this paper. Through the retransmission deferral timing function and the geo-position information, a broadcast suppression sector can be formed and distance assisted packet forwarding can be realized. By prohibiting packet forwarding in the suppression sector, large amount of packet forwarding can be eliminated, especially when in network caching is used in an ICN network. In this way, our method can reduce the interference of broadcasting data in the wireless environment, which may potentially increase the throughput of the whole network. Our simulation results show that the proposed method can greatly reduce the total number of packets forwarded in the network, while keeping a good delivery ratio of the requested data and a low data response time.

There are still some assumptions in our method, such as random distribution of vehicles. Our next step is to evaluate our method more thoroughly and improve our method by considering the distribution of vehicles along certain roads and the density of vehicles on the road. In our current results, we could see the importance of broadcast suppression at higher node densities. By estimating the density of vehicles, for example like in [25], it may be possible to adjust the angle used for the broadcast suppression sector to further improve performance for a wide range of scenarios. While the focus in this paper has been on the forwarding of interest messages, we also want to study the impact of utilizing caching in the network, and in particular infrastructure assisted caching and forwarding.

\section{$7 \quad$ References}

1. Ahlgren, B., Dannewitz, C., Imbrenda, C. and et al: A Survey of Information-centric Networking. IEEE Communications Magazine, July 2012.

2. L. Zhang, A. Afanasyev, J. Burke, V. Jacobson and et al: Named Data Networking. ACM SIGCOMM Computer Communication Review (CCR), July 2014.

3. Sharef, B.T., Alsaqour, R.A., and Ismail, M.: Vehicular Communication Ad Hoc Routing Protocols: A Survey. Journal of Network and Computer Applications, 40 (2014), pp. 363396.

4. Daraghmi, Y.A., Yi, C.W. and Stojmenovic, I.: Forwarding Methods in Dissemination and Routing Protocols for Vehicular Ad Hoc Networks. IEEE Network, Nov./Dec. 2013.

5. Wang, J., et al: DMND: Collecting Data from Mobiles Using Name Data. IEEE Vehicular Networking Conference, 2010.

6. Wang, L., et al: Rapid traffic information dissemination using named data. ACM MobiHoc NoM Workshop, 2012.

7. Amadeo, M., Campolo, C., and Molinaro, A.: Enhancing Content - Centric Networking for Vehicular Environments. Computer Networks, 57(16), November 2013.

8. NS-3 based Named Data Networking (NDN) simulator. http://ndnsim.net/2.0/.

9. NS-3: A Discrete-event Network Simulator. http://www.nsnam.org/

10. OpenStreetMap. http://www.openstreetmap.org.

11. Simulation of Urban Mobility. http://sumo.sourceforge.net

12. Perkins, C.E. and Royer, E.M.: Ad-hoc on demand distance vector routing. In: $2^{\text {nd }}$ IEEE Workshop on Mobile Computing Systems and Applications, pp. 90-100, Feb. 1999. 
13. Johnson, D.B. and Maltz, D.A.: Dynamic source routing in ad hoc wireless networks. Mobile Computing, vol. 353 .

14. Liu, G., Lee, B.S., Seet, B.C., Foh, C.H., Wong, K.J. and Lee, K.K.: A routing strategy for metropolis vehicular communications. In: International Conference on Information Networking (ICOIN), pp. 134-143, 2004.

15. Füßler, H., Mauve, M., Hartenstein, H., Kasemann, M. and Vollmer, D.: Location based routing for vehicular ad-hoc networks. ACM SIGMOBILE Mobile Computing and Communications Review (MC2R), vol. 7, no. 1, pp. 47-49, Jan. 2003.

16. Santos, R.A., Edwards, A., Edwards, R. and Seed, L.: Performance evaluation of routing protocols in vehicular ad hoc networks. The International Journal of Ad Hoc and Ubiquitous Computing, vol. 1, no. 1/2, pp. 80-91, 2005.

17. Lochert, C., Mauve, M., Füßler, H. and Hartenstein, H.: Geographic routing in city scenarios. ACM SIGMOBILE Mobile Computing and Communications Review (MC2R), vol. 9, no. 1, pp. 69-72, Jan. 2005.

18. Korkmaz, G., Ekici, E., Özgüner, F. and Özgüner, Ü.: Urban multi-hop broadcast protocol for inter-vehicle communication systems. In: ACM International Workshop on Vehicular Ad Hoc Networks, pp. 76-85, 2004.

19. Little, T.D.C. and Agarwal, A.: An information propagation scheme for VANETs. In: $8^{\text {th }}$ International IEEE Conference on Intelligent Transportation Systems (ITSC 2005), 2005.

20. Karp B., Kung H. T.: GPSR: Greedy perimeter stateless routing for wireless networks. In: $6^{\text {th }}$ annual international conference on Mobile computing and networking. ACM, 2000: 243254.

21. Pentikousis, K., Ohlman, B., Corujo, D., Boggia, G., Tyson, G., Davies, E., Molinaro, A., Eum, S.: Information-Centric Networking: Baseline Scenarios, RFC7476, https://tools.ietf.org/html/rfc7476, March 2015.

22. Jacobson, V., Smetters, D. K. et al: Networking Named Content. In: $5^{\text {th }}$ International Conference on Emerging Networking Experiments and Technologies, Dec. 2009.

23. Grassi, G., Pesavento, D., Pau, G., Vuyyuru, R., Wakikawa, R., and Zhang, L: VANET via Named Data Networking. INFOCOM Workshop on Name Oriented Mobility (NOM), Toronto, Canada, April-May 2014.

24. Grassi, G., Pesavento, D., Pau, G., Zhang, L, Fdida, S.: Navigo: Interest Forwarding by Geolocations in Vehicular Named Data Networking. In: IEEE World of Wireless Mobile and Multimedia Networks (WoWMoM), Boston, USA, June 2015.

25. Wennerström, H., Rohner, C.: Towards Even Coverage Monitoring with Opportunistic Sensor Networks. In: Proc. of ACM MobiCom Workshop on Challenged Networks (CHANTS), New York, USA, October 2016. 